\title{
ERROR ESTIMATORS FOR NONCONFORMING FINITE ELEMENT APPROXIMATIONS OF THE STOKES PROBLEM
}

\author{
ENZO DARI, RICARDO DURÁN, AND CLAUDIO PADRA
}

\begin{abstract}
In this paper we define and analyze a posteriori error estimators for nonconforming approximations of the Stokes equations. We prove that these estimators are equivalent to an appropriate norm of the error. For the case of piecewise linear elements we define two estimators. Both of them are easy to compute, but the second is simpler because it can be computed using only the right-hand side and the approximate velocity. We show how the first estimator can be generalized to higher-order elements. Finally, we present several numerical examples in which one of our estimators is used for adaptive refinement.
\end{abstract}

\section{INTRODUCTION}

In recent years there has been considerable interest in the development of computable a posteriori error estimates in the finite element method (see $[3,4,5$, and 12] and references therein). The object of this work is to define and analyze a posteriori error estimators for nonconforming finite element approximations of the Stokes equations.

The use of nonconforming elements for the Stokes problem is motivated by the fact that standard low-order elements do not satisfy the inf-sup condition. In contrast, the nonconforming linear elements of Crouzeix and Raviart [8] and the quadratic elements of Fortin and Soulie [10] do satisfy that stability condition and therefore provide optimal order of convergence.

In the conforming case there are several ways to define error estimators by using the residual equation. In particular, for the Stokes problem, Verfürth [16] and Bank and Welfert $[6,7]$ introduced several error estimators and proved that they are equivalent to the energy norm of the error.

In the nonconforming case there is an estimator introduced by Verfürth [17] who proved the equivalence of it with the norm of the error but neglecting the consistency terms in the error equations. However, these terms are not in general of higher order, and therefore the estimators have to contain a term related to them, which are the jumps of the tangential derivatives of the approximate solution, as we show in this paper. Indeed, these terms are very important and cannot be neglected, as is shown by our Theorem 3.3. Otherwise, it would be possible to construct an estimator equivalent to the error which would depend only on the right-hand side $\mathbf{f}$. This estimator would be of order $h$ whenever

Received by the editor August 5, 1992 and, in revised form, March 14, 1994.

1991 Mathematics Subject Classification. Primary 65N30, 65N15, 65N50, 76D07.

The first and second authors are members of CONICET, Argentina. 
$f$ is regular, and therefore it cannot be equivalent to the norm of the error in problems with singular solutions.

In [9] we considered the simpler case of nonconforming approximations of a scalar second-order elliptic problem, and we introduced a technique which allowed us to define two error estimators which are equivalent to the error. In this paper we show that, with appropriate modifications, the ideas in [9] can be extended to the Stokes problem. We define two error estimators based on suitable evaluations of the residual and prove that they are equivalent to the energy norm of the error.

In $\S 2$ we introduce some notations and recall the Crouzeix-Raviart elements. In $\S 3$ we define the error estimators and prove their equivalence with the error. Section 4 deals with the extension to the quadratic elements of Fortin and Soulie and finally, in $\S 5$ we present some numerical computations in which one of our error estimators is used for adaptive refinement.

\section{Preliminaries and Notations}

Given a simply connected polygon $\Omega \subset \mathbf{R}^{2}$, we consider the Stokes problem

$$
\begin{cases}-\Delta \mathbf{u}+\nabla p=\mathbf{f} & \text { in } \Omega, \\ \operatorname{div} \mathbf{u}=0 & \text { in } \Omega, \\ \mathbf{u}=0 & \text { on } \partial \Omega,\end{cases}
$$

where $\mathbf{u}$ stands for the velocity and $p$ for the pressure. The weak formulation appropriate for mixed methods is then:

Find $\mathbf{u} \in \mathbf{H}_{0}^{1}(\Omega)$ and $p \in L_{0}^{2}(\Omega)$ such that

$$
\begin{cases}\int_{\Omega} \nabla \mathbf{u}: \nabla \mathbf{v}-\int_{\Omega} p \operatorname{div} \mathbf{v}=\int_{\Omega} \mathbf{f} \cdot \mathbf{v}, & \forall \mathbf{v} \in \mathbf{H}_{0}^{1}(\Omega), \\ \int_{\Omega} q \operatorname{div} \mathbf{u}=0, & \forall q \in L_{0}^{2}(\Omega),\end{cases}
$$

where, $\mathbf{H}_{0}^{1}(\Omega)=H_{0}^{1}(\Omega) \times H_{0}^{1}(\Omega), L_{0}^{2}(\Omega)=\left\{q \in L^{2}(\Omega): \int_{\Omega} q=0\right\}, \nabla \mathbf{u}$ is the matrix $\left(\frac{\partial u_{i}}{\partial x_{j}}\right)$, and we use the standard notation for the contraction of two matrices $A$ and $B$, i.e.,

$$
A: B=\sum_{i, j=1}^{2} A_{i j} B_{i j},
$$

and for Sobolev spaces. Also, $\|\cdot\|_{j, D}$ and $|\cdot|_{j, D}$ will denote the usual $j$ norm and seminorm, respectively, on $D$, and when $D=\Omega$ the subscript $D$ will be supressed.

Assume that we have a family $\left\{\mathscr{T}^{k}\right\}$ of regular triangulations of $\Omega$ such that any two triangles in $\mathscr{T}^{k}$ share at most a vertex or an edge. The CrouzeixRaviart nonconforming finite element spaces are defined by

$$
\begin{aligned}
\mathbf{V}^{k}=\left\{\mathbf{v} \in L^{2}(\Omega) \times L^{2}(\Omega):\left.\mathbf{v}\right|_{T} \in \mathscr{P}_{1} \times \mathscr{P}_{1}, \forall T \in \mathscr{T}^{k},\right. \\
\mathbf{v} \text { is continuous at midpoints of edges and } 0 \text { at midpoints } \\
\text { of edges contained in } \partial \Omega\}
\end{aligned}
$$

and

$$
Q^{k}=\left\{q \in L_{0}^{2}(\Omega):\left.q\right|_{T} \in \mathscr{P}_{0}, \forall T \in \mathscr{T}^{k}\right\}
$$

(where $\mathscr{P}_{r}$ denotes the space of polynomials of degree not greater than $r$ ). 
The approximate solution $\left(\mathbf{u}^{k}, p^{k}\right) \in \mathbf{V}^{k} \times Q^{k}$ of problem $(2.1)$ is defined by

$$
\begin{aligned}
& \sum_{T \in \mathscr{T}^{k}}\left\{\int_{T} \nabla \mathbf{u}^{k}: \nabla \mathbf{v}-\int_{T} p^{k} \operatorname{div} \mathbf{v}\right\}=\int_{\Omega} \mathbf{f} \cdot \mathbf{v}, \forall \mathbf{v} \in \mathbf{V}^{k}, \\
& \sum_{T \in \mathscr{T}^{k}} \int_{T} q \operatorname{div} \mathbf{u}^{k}=0, \forall q \in Q^{k} .
\end{aligned}
$$

Note that the second equation means that for every $T \in \mathscr{T}^{k}, \operatorname{div}\left(\left.\mathbf{u}^{k}\right|_{T}\right)=0$.

In the analysis of the error estimators we will also use the standard conforming space

$$
\mathbf{M}^{k}=\left\{\mathbf{v} \in \mathbf{H}^{1}(\Omega):\left.\mathbf{v}\right|_{T} \in \mathscr{P}_{1} \times \mathscr{P}_{1}, \forall T \in \mathscr{T}^{k}\right\} .
$$

We end this section with some notation. For a vector function $\mathbf{w}=\left(\begin{array}{l}w_{1} \\ w_{2}\end{array}\right)$ we define the matrix curl $\mathbf{w}$ by

$$
\text { curl } \mathbf{w}=\left(\begin{array}{ll}
-\frac{\partial w_{1}}{\partial x_{2}} & \frac{\partial w_{1}}{\partial x_{1}} \\
-\frac{\partial w_{2}}{\partial x_{2}} & \frac{\partial w_{2}}{\partial x_{1}}
\end{array}\right),
$$

and for a matrix $A$ the divergence of $A$ is the vector,

$$
\operatorname{div} A=\left(\begin{array}{l}
\frac{\partial A_{11}}{\partial x_{1}}+\frac{\partial A_{12}}{\partial x_{2}} \\
\frac{\partial A_{21}}{\partial x_{1}}+\frac{\partial A_{22}}{\partial x_{2}}
\end{array}\right) .
$$

Observe that with these notations we have

$$
\int_{D} \operatorname{div} A \cdot \mathbf{w}=-\int_{D} A: \nabla \mathbf{w}+\int_{\partial D} A \mathbf{n} \cdot \mathbf{w}
$$

for any $A$ and $\mathbf{w}$ with the appropriate regularity, where $\mathbf{n}$ is the outward normal to $\partial D$.

Also,

$$
\int_{D} \nabla \mathbf{v}: \operatorname{curl} \mathbf{w}=\int_{\partial D} \nabla \mathbf{v t} \cdot \mathbf{w}
$$

for any $\mathbf{v} \in \mathbf{H}^{1}(D)$ and $\mathbf{w} \in \mathbf{H}^{1}(D)$, where $\mathbf{t}$ is the tangent to $\partial D$.

\section{ERROR ESTIMATORS}

In this section we introduce the error estimators and prove their equivalence with the error.

To define the estimators we need to introduce some jumps associated with the discrete solution $\left(\mathbf{u}^{k}, p^{k}\right) \in \mathbf{V}^{k} \times Q^{k}$. Given an interior edge $l$, we choose an arbitrary normal direction $\mathbf{n}_{l}$ and denote with $T_{\text {in }}$ and $T_{\text {out }}$ the two triangles sharing this edge, with $\mathbf{n}_{l}$ pointing out of $T_{\text {in }}$ as in Fig. 3.1.

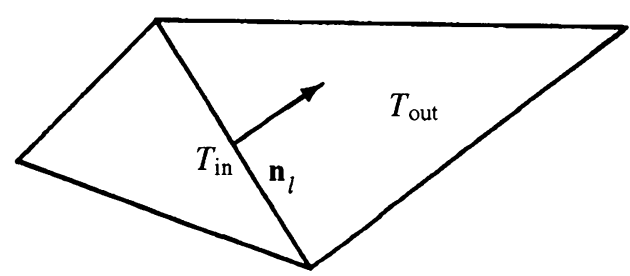

FigURE 3.1 
If $\mathbf{n}_{l}=\left(\begin{array}{l}n_{1} \\ n_{2}\end{array}\right)$, we define the tangent $\mathbf{t}_{l}=\left(\begin{array}{c}-n_{2} \\ n_{1}\end{array}\right)$. When $l$ is a boundary edge, $\mathbf{n}_{l}$ is the outward normal.

We define

$$
\llbracket\left(\nabla \mathbf{u}^{k}-p^{k} \mathbf{I}\right) \mathbf{n}_{l} \rrbracket_{l}=\left[\nabla\left(\left.\mathbf{u}^{k}\right|_{T_{\text {out }}}\right)-\left.p^{k}\right|_{T_{\text {out }}} \mathbf{I}\right] \mathbf{n}_{l}-\left[\nabla\left(\left.\mathbf{u}^{k}\right|_{T_{\text {in }}}\right)-\left.p^{k}\right|_{T_{\text {in }}} \mathbf{I} \mathbf{n}_{l},\right.
$$

where $I$ is the identity matrix, and

$$
\llbracket \nabla \mathbf{u}^{k} \mathbf{t}_{l} \rrbracket_{l}=\nabla\left(\mathbf{u}^{k} \mid T_{\text {out }}\right) \mathbf{t}_{l}-\nabla\left(\left.\mathbf{u}^{k}\right|_{T_{\text {in }}}\right) \mathbf{t}_{l} .
$$

Let $E_{I}$ be the set of interior edges of $\mathscr{T}^{k}$, and for an element $T$, let $E_{T}$ be the set of edges of $T$.

Now set

$$
\mathbf{J}_{l, n}= \begin{cases}\llbracket\left(\nabla \mathbf{u}^{k}-p^{k} \mathbf{I}\right) \mathbf{n}_{l} \rrbracket_{l} & \text { if } l \in E_{I}, \\ 0 & \text { if } l \subset \partial \Omega\end{cases}
$$

and

$$
\mathbf{J}_{l, t}= \begin{cases}\llbracket \nabla \mathbf{u}^{k} \mathbf{t}_{l} \rrbracket_{l} & \text { if } l \in E_{I}, \\ 2 \nabla \mathbf{u}^{k} \mathbf{t}_{l} & \text { if } l \subset \partial \Omega\end{cases}
$$

With these notations we introduce the local error estimator $\eta_{T}$ defined by

$$
\eta_{T}^{2}=|T|\|\mathbf{f}\|_{0, T}^{2}+\frac{1}{2} \sum_{l \in E_{T}}|l|^{2}\left(\left|\mathbf{J}_{l, n}\right|^{2}+\left|\mathbf{J}_{l, t}\right|^{2}\right)
$$

and the global one,

$$
\eta=\left(\sum_{T} \eta_{T}^{2}\right)^{\frac{1}{2}} .
$$

Let $\mathbf{e}=\mathbf{u}-\mathbf{u}^{k}$ and $\varepsilon=p-p^{k}$ be the errors in velocity and pressure, respectively.

For a piecewise regular vector function $\mathbf{v}$ we define the discrete gradient as the $L^{2}$-matrix defined by

$$
\left.\nabla_{k} \mathbf{v}\right|_{T}=\nabla\left(\left.\mathbf{v}\right|_{T}\right)
$$

We will use the following error equation, which is obtained subtracting (2.3) from (2.2) for any $\mathbf{v} \in \mathbf{H}_{0}^{1}(\Omega) \cap \mathbf{V}^{k}$ :

$$
\int_{\Omega} \nabla_{k} \mathbf{e}: \nabla \mathbf{v}-\int_{\Omega} \varepsilon \operatorname{div} \mathbf{v}=0, \quad \forall \mathbf{v} \in \mathbf{H}_{0}^{1}(\Omega) \cap \mathbf{V}^{k} .
$$

For a function $\mathbf{v} \in \mathbf{H}^{1}(\Omega)$ we take $\mathbf{v}^{I} \in \mathbf{M}^{k}$ as a suitable interpolation of $\mathbf{v}$ satisfying

$$
\left\|\mathbf{v}-\mathbf{v}^{I}\right\|_{0, T} \leq C|T|^{\frac{1}{2}}|\mathbf{v}|_{1, \widetilde{T}}
$$

and

$$
\left\|\mathbf{v}-\mathbf{v}^{I}\right\|_{0, l} \leq C|l|^{\frac{1}{2}}|\mathbf{v}|_{1, \widetilde{T}} \quad \text { for } l \in E_{T},
$$

where $\tilde{T}$ is the union of all the elements sharing a vertex with $T$. We recall that if $\mathbf{v} \in \mathbf{H}_{0}^{1}(\Omega)$, then $\mathbf{v}^{l}$ can be taken in $\mathbf{H}_{0}^{1}(\Omega) \cap \mathbf{M}^{k}$ (see for example [14] for the construction of this kind of interpolation). Here and hereafter the letter $C$ denotes a generic constant which depends only on the minimum angle of the triangulation. 
First we will show that the norm of the error,

$$
\|\varepsilon\|_{0}+\left\|\nabla_{k} \mathbf{e}\right\|_{0}
$$

is dominated by the estimator. This will be a consequence of two lemmas. The first shows that the error in pressure is dominated by the error in velocity plus the estimator, and the second bounds the velocity error by a constant times the estimator.

Lemma 3.1. The following estimate holds:

$$
\|\varepsilon\|_{0} \leq C\left\{\eta+\left\|\nabla_{k} \mathbf{e}\right\|_{0}\right\}
$$

Proof. Since $\varepsilon \in L_{0}^{2}(\Omega)$, there exists $\mathbf{v} \in \mathbf{H}_{0}^{1}(\Omega)$ [11] such that

$$
\|\varepsilon\|_{0} \leq C \frac{\int_{\Omega} \varepsilon \operatorname{div} \mathbf{v}}{|\mathbf{v}|_{1}}
$$

Now, since $\mathbf{v}^{I} \in \mathbf{M}^{k} \cap \mathbf{H}_{0}^{1}(\Omega) \subset \mathbf{V}^{k} \cap \mathbf{H}_{0}^{1}(\Omega)$, we can use the error equation (3.1) to obtain

$$
\begin{aligned}
\int_{\Omega} \varepsilon \operatorname{div} \mathbf{v} & =\int_{\Omega} \varepsilon \operatorname{div}\left(\mathbf{v}-\mathbf{v}^{I}\right)+\int_{\Omega} \varepsilon \operatorname{div} \mathbf{v}^{I} \\
& =\int_{\Omega} \varepsilon \operatorname{div}\left(\mathbf{v}-\mathbf{v}^{I}\right)+\int_{\Omega} \nabla_{k} \mathbf{e}: \nabla \mathbf{v}^{I} \\
& =\int_{\Omega} \varepsilon \operatorname{div}\left(\mathbf{v}-\mathbf{v}^{I}\right)-\int_{\Omega} \nabla_{k} \mathbf{e}: \nabla\left(\mathbf{v}-\mathbf{v}^{I}\right)+\int_{\Omega} \nabla_{k} \mathbf{e}: \nabla \mathbf{v} .
\end{aligned}
$$

Thus, integration by parts in each element yields

$$
\begin{aligned}
\int_{\Omega} \varepsilon \operatorname{div} \mathbf{v}= & \sum_{T \in \mathscr{T}^{k}}\left\{\int_{T}(-\nabla p+\Delta \mathbf{u}) \cdot\left(\mathbf{v}-\mathbf{v}^{I}\right)\right. \\
& \left.\quad+\int_{\partial T}\left(\nabla_{k} \mathbf{u}^{k}-p^{k} \mathbf{I}\right) \mathbf{n} \cdot\left(\mathbf{v}-\mathbf{v}^{I}\right)\right\}+\int_{\Omega} \nabla_{k} \mathbf{e}: \nabla \mathbf{v} \\
= & \sum_{T}\left\{-\int_{T} \mathbf{f} \cdot\left(\mathbf{v}-\mathbf{v}^{I}\right)-\frac{1}{2} \sum_{l \in E_{T}} \int_{l} \mathbf{J}_{l, n} \cdot\left(\mathbf{v}-\mathbf{v}^{I}\right)\right\}+\int_{\Omega} \nabla_{k} \mathbf{e}: \nabla \mathbf{v} .
\end{aligned}
$$

Now, using the Schwarz inequality, (3.2) and (3.3), we obtain

$$
\int_{\Omega} \varepsilon \operatorname{div} \mathbf{v} \leq C\left(\eta+\left\|\nabla_{k} \mathbf{e}\right\|_{0}\right)|\mathbf{v}|_{1},
$$

which together with (3.4) proves the lemma.

Lemma 3.2. The following estimate holds:

$$
\left\|\nabla_{k} \mathbf{e}\right\|_{0} \leq C \eta
$$

Proof. First we decompose the error as

$$
\nabla_{k} \mathbf{e}=\nabla \mathbf{r}-q I+\operatorname{curl} \mathbf{s}
$$

with $\mathbf{r} \in \mathbf{H}_{0}^{1}(\Omega), q \in L_{0}^{2}(\Omega)$ and $\mathbf{s} \in \mathbf{H}^{1}(\Omega)$ satisfying

$$
\|\mathbf{r}\|_{1}+\|\mathbf{s}\|_{1} \leq C\left\|\nabla_{k} \mathbf{e}\right\|_{0}
$$

and

$$
\operatorname{div} \mathbf{r}=0
$$


This decomposition can be obtained as follows. Let $\mathbf{r} \in \mathbf{H}_{0}^{1}(\Omega)$ and $q \in L_{0}^{2}$ be the solution of the Stokes problem with right-hand side $-\operatorname{div}\left(\nabla_{k} \mathbf{e}\right) \in \mathbf{H}^{-1}(\Omega)$, i.e.,

$$
\left\{\begin{array}{l}
-\Delta \mathbf{r}+\nabla q=-\operatorname{div}\left(\nabla_{k} \mathbf{e}\right) \\
\operatorname{div} \mathbf{r}=0
\end{array}\right.
$$

From standard a priori estimates we know that

$$
\|\mathbf{r}\|_{1}+\|q\|_{0} \leq C\left\|\nabla_{k} \mathbf{e}\right\|_{0} .
$$

On the other hand, the first equation in (3.8) can be written as

$$
\operatorname{div}\left(\nabla \mathbf{r}-q \mathbf{I}-\nabla_{k} \mathbf{e}\right)=0 .
$$

Therefore, there exists $\mathbf{s} \in \mathbf{H}^{1}(\Omega)$, with integral zero, such that (3.5) holds, and the bound for $\|\mathbf{s}\|_{1}$ follows from (3.9) and (3.5).

Now we estimate the velocity error using the decomposition (3.5). First observe that

$$
\int_{\Omega} \nabla_{k} \mathbf{e}: q \mathbf{I}=\sum_{T} \int_{T} q \operatorname{div} \mathbf{e}=0
$$

because $\operatorname{div}\left(\left.\mathbf{e}\right|_{T}\right)=0$. Therefore, since $\operatorname{div} \mathbf{r}=0$, we have

$$
\left\|\nabla_{k} \mathbf{e}\right\|_{0}^{2}=\int_{\Omega} \nabla_{k} \mathbf{e}: \nabla \mathbf{r}-\int_{\Omega} \varepsilon \operatorname{div} \mathbf{r}+\int_{\Omega} \nabla_{k} \mathbf{e}: \text { curl } \mathbf{s} .
$$

Using the error equation (3.1) for $\mathbf{v}=\mathbf{r}^{I} \in \mathbf{H}_{0}^{1} \cap \mathbf{V}^{k}$ and the orthogonality relation

$$
\int_{\Omega} \nabla_{k} \mathbf{e}: \operatorname{curl}^{I}=0,
$$

which is known [1] and easy to verify, we obtain from (3.10) that

$$
\begin{aligned}
\left\|\nabla_{k} \mathbf{e}\right\|_{0}^{2} & =\int_{\Omega} \nabla_{k} \mathbf{e}: \nabla\left(\mathbf{r}-\mathbf{r}^{I}\right)-\int_{\Omega} \varepsilon \operatorname{div}\left(\mathbf{r}-\mathbf{r}^{I}\right)+\int_{\Omega} \nabla_{k} \mathbf{e}: \operatorname{curl}\left(\mathbf{s}-\mathbf{s}^{I}\right) \\
& =\int_{\Omega}\left(\nabla_{k} \mathbf{e}-\varepsilon \mathbf{I}\right): \nabla\left(\mathbf{r}-\mathbf{r}^{I}\right)+\int_{\Omega} \nabla_{k} \mathbf{e}: \operatorname{curl}\left(\mathbf{s}-\mathbf{s}^{I}\right) .
\end{aligned}
$$

Integrating by parts in each element, we obtain

$$
\begin{aligned}
\left\|\nabla_{k} \mathbf{e}\right\|_{0}^{2} & =\sum_{T}\left\{\int_{T} \mathbf{f} \cdot\left(\mathbf{r}-\mathbf{r}^{I}\right)-\int_{\partial T}\left(\nabla_{k} \mathbf{u}^{k}-p^{k} \mathbf{I}\right) \mathbf{n} \cdot\left(\mathbf{r}-\mathbf{r}^{I}\right)-\int_{\partial T} \nabla_{k} \mathbf{u}^{k} \mathbf{t} \cdot\left(\mathbf{s}-\mathbf{s}^{I}\right)\right\} \\
& =\sum_{T}\left\{\int_{T} \mathbf{f} \cdot\left(\mathbf{r}-\mathbf{r}^{I}\right)+\frac{1}{2} \sum_{l \in E_{T}}\left[\int_{l} \mathbf{J}_{l, n} \cdot\left(\mathbf{r}-\mathbf{r}^{I}\right)+\int_{l} \mathbf{J}_{l, t} \cdot\left(\mathbf{s}-\mathbf{s}^{I}\right)\right]\right\}
\end{aligned}
$$

(where $\mathbf{t}$ denotes the tangent to $\partial T$ ), and applying the Schwarz inequality, (3.2), (3.3) and (3.6), we obtain the lemma.

As an immediate consequence of the two lemmas above we have the following

Theorem 3.1. There exists a constant $C$ such that

$$
\|\varepsilon\|_{0}+\left\|\nabla_{k} \mathbf{e}\right\|_{0} \leq C \eta .
$$


In the next theorem we show that the estimator is essentially dominated by the error. Indeed, we extend to our case the technique introduced in [16] for conforming elements and prove that the estimator is bounded by a constant times the error plus a higher-order term which depends on local regularity of the right-hand side.

Theorem 3.2. Let $\mathbf{f}_{T}=\frac{1}{|T|} \int_{T} \mathbf{f}$. Then there exists a constant $C$ such that

$$
\eta \leq C\left\{\|\varepsilon\|_{0}+\left\|\nabla_{k} \mathbf{e}\right\|_{0}+\left(\sum_{T}|T|\left\|\mathbf{f}-\mathbf{f}_{T}\right\|_{0, T}^{2}\right)^{\frac{1}{2}}\right\} .
$$

Proof. Proceeding as in Lemma 3.2, we see that

$$
\begin{aligned}
\int_{\Omega} \nabla_{k} \mathbf{e}:(\nabla \mathbf{r}+\operatorname{curl} \mathbf{s})-\int_{\Omega} \varepsilon \operatorname{div} \mathbf{r} \\
=\sum_{T}\left\{\int_{T} \mathbf{f}_{T} \cdot \mathbf{r}+\frac{1}{2} \sum_{l \in E_{T}}\left[\int_{l} \mathbf{J}_{l, n} \cdot \mathbf{r}+\int_{l} \mathbf{J}_{l, t} \cdot \mathbf{s}\right]+\int_{T}\left(\mathbf{f}-\mathbf{f}_{T}\right) \cdot \mathbf{r}\right\}
\end{aligned}
$$

for every $\mathbf{r} \in \mathbf{H}_{0}^{1}(\Omega)$ and every $\mathbf{s} \in \mathbf{H}^{1}(\Omega)$.

Now we choose $\mathbf{r}$ and $\mathbf{s}$ such that

$$
\begin{aligned}
& \int_{T} \mathbf{f}_{T} \cdot \mathbf{r}=|T|^{2}\left|\mathbf{f}_{T}\right|^{2}, \quad \forall T \in \mathscr{T}^{k}, \\
& \int_{l} \mathbf{J}_{l, n} \cdot \mathbf{r}=|l|^{2}\left|\mathbf{J}_{l, n}\right|^{2}, \quad \forall l \in E_{I}, \\
& \int_{l} \mathbf{J}_{l, t} \cdot \mathbf{s}=|l|^{2}\left|\mathbf{J}_{l, t}\right|^{2}, \quad \forall l \in E_{I}, \forall l \subset \partial \Omega
\end{aligned}
$$

and

$$
|\mathbf{r}|_{1, T}+|\mathbf{s}|_{1, T} \leq C \eta_{T}
$$

These functions can be taken in the following way:

$$
\mathbf{r}=\alpha_{T} \mathbf{f}_{T} b_{T}+\sum_{l \in E_{T}} \beta_{l} \mathbf{J}_{l, n} \varphi_{l}
$$

and

$$
\mathbf{s}=\sum_{l \in E_{T}} \gamma_{l} \mathbf{J}_{l, t} \varphi_{l}
$$

where $b_{T}$ is a usual bubble function on $T$ (i.e., a cubic polynomial vanishing on $\partial T), \varphi_{l}$ is the standard quadratic basis function which takes the value 1 at the midpoint of $l$ and 0 at the other nodes, and $\alpha_{T}, \beta_{l}$ and $\gamma_{l}$ are scalars chosen such that conditions (3.13) are satisfied. The estimate (3.14) follows from standard scaling arguments. 
From (3.12) and (3.13) we get

$$
\begin{aligned}
\int_{\Omega} \nabla_{k} \mathbf{e}:(\nabla \mathbf{r}+\operatorname{curl} \mathbf{s})-\int_{\Omega} \varepsilon \operatorname{div} \mathbf{r} & \\
= & \sum_{T}\left\{|T|^{2}|\mathbf{f}|_{T}^{2}+\frac{1}{2} \sum_{l \in E_{T}}|l|^{2}\left(\left|\mathbf{J}_{l, n}\right|^{2}+\left|\mathbf{J}_{l, t}\right|^{2}\right)+\int_{T}\left(\mathbf{f}-\mathbf{f}_{T}\right) \cdot \mathbf{r}\right\} \\
= & \sum_{T}\left\{|T|\|\mathbf{f}\|_{0, T}^{2}+\frac{1}{2} \sum_{l \in E_{T}}|l|^{2}\left(\left|\mathbf{J}_{l, n}\right|^{2}+\left|\mathbf{J}_{l, t}\right|^{2}\right)\right. \\
& \left.+\int_{T}\left(\mathbf{f}-\mathbf{f}_{T}\right) \cdot \mathbf{r}-|T|\left\|\mathbf{f}-\mathbf{f}_{T}\right\|_{0, T}^{2}\right\} \\
= & \eta^{2}+\sum_{T}\left\{\int_{T}\left(\mathbf{f}-\mathbf{f}_{T}\right) \cdot\left(\mathbf{r}-\mathbf{r}_{T}\right)-|T|\left\|\mathbf{f}-\mathbf{f}_{T}\right\|_{0, T}^{2}\right\} .
\end{aligned}
$$

Therefore, using the Schwarz inequality, (3.14), the known estimate $\left\|\mathbf{r}-\mathbf{r}_{T}\right\|_{0, T}$ $\leq C|T|^{\frac{1}{2}}|\mathbf{r}|_{1, T}$ and $|T|^{\frac{1}{2}}\left\|\mathbf{f}-\mathbf{f}_{T}\right\|_{0, T} \leq \eta_{T}$, we obtain

$$
\begin{aligned}
\eta^{2} \leq & \left(\|\varepsilon\|_{0}+\left\|\nabla_{k} \mathbf{e}\right\|_{0}\right)\left(|\mathbf{r}|_{1}+|\mathbf{s}|_{1}\right) \\
& +C \sum_{T}\left\|\mathbf{f}-\mathbf{f}_{T}\right\|_{0, T}|T|^{\frac{1}{2}}\left(|\mathbf{r}|_{1, T}+\eta_{T}\right) \\
\leq & C\left\{\|\varepsilon\|_{0}+\left\|\nabla_{k} \mathbf{e}\right\|_{0}+\left(\sum_{T}\left\|\mathbf{f}-\mathbf{f}_{T}\right\|_{0, T}^{2}|T|\right)^{\frac{1}{2}}\right\} \eta,
\end{aligned}
$$

and the theorem is proved.

We now introduce an estimator which is equivalent to $\eta$ and simpler to compute.

To do this, we show that the terms corresponding to $\mathbf{J}_{l, n}$ can be eliminated.

Lemma 3.3. Given an edge $l \in E_{I}$, the following inequality holds (we use the notation of Fig. 3.1):

$$
\left|\mathbf{J}_{l, n}\right|^{2}|l|^{2} \leq\|\mathbf{f}\|_{0, T_{\text {in }}}^{2} \frac{\left|T_{\text {in }}\right|}{3}+\|\mathbf{f}\|_{0, T_{\text {out }}}^{2} \frac{\left|T_{\text {out }}\right|}{3} .
$$

Proof. Let $\psi_{l}$ be the basis function associated with the midpoint of $l$, that is, $\psi_{l}$ is one at this midpoint and vanishes at all other nodes.

Taking $\mathbf{v}^{1}=\left(\begin{array}{c}\psi_{l} \\ 0\end{array}\right)$ and $\mathbf{v}^{2}=\left(\begin{array}{c}0 \\ \psi_{l}\end{array}\right)$ as test functions in (2.3), and integrating by parts, we obtain

$$
\begin{aligned}
\int_{T_{\text {in }} \cup T_{\text {out }}} \mathbf{f} \cdot \mathbf{v}^{i} & =\int_{T_{\text {in }}} \nabla_{k} \mathbf{u}^{k}: \nabla \mathbf{v}^{i}-\int_{T_{\text {in }}} p^{k} \operatorname{div} \mathbf{v}^{i}+\int_{T_{\text {out }}} \nabla_{k} \mathbf{u}^{k}: \nabla \mathbf{v}^{i}-\int_{T_{\text {out }}} p^{k} \operatorname{div} \mathbf{v}^{i} \\
& =\int_{\partial T_{\text {in }}}\left(\nabla \mathbf{u}^{k}-p^{k} \mathbf{I}\right) \mathbf{n}_{l} \cdot \mathbf{v}^{i}-\int_{\partial T_{\text {out }}}\left(\nabla \mathbf{u}^{k}-p^{k} \mathbf{I}\right) \mathbf{n}_{l} \cdot \mathbf{v}^{i} \\
& =-\int_{l} \mathbf{J}_{l, n} \cdot \mathbf{v}^{i} .
\end{aligned}
$$


Therefore, denoting by $J_{l, n}^{i}$ and $f^{i}, i=1,2$, the two components of $\mathbf{J}_{l, n}$ and $\mathbf{f}$, respectively, we have

$$
-J_{l, n}^{i}|l|=\int_{T_{\text {in }} \cup T_{\text {out }}} f^{i} \psi_{l}
$$

and so

$$
\begin{aligned}
\left|\mathbf{J}_{l, n}\right||l| & \leq\|\mathbf{f}\|_{0, T_{\text {in }}}\left\|\psi_{l}\right\|_{0, T_{\text {in }}}+\|\mathbf{f}\|_{0, T_{\text {out }}}\left\|\psi_{l}\right\|_{0, T_{\text {out }}} \\
& =\|\mathbf{f}\|_{0, T_{\text {in }}}\left(\frac{\left|T_{\text {in }}\right|}{3}\right)^{\frac{1}{2}}+\|\mathbf{f}\|_{0, T_{\text {out }}}\left(\frac{\left|T_{\text {out }}\right|}{3}\right)^{\frac{1}{2}},
\end{aligned}
$$

which concludes the proof.

Consequently, we define the estimator $\tilde{\eta}$ by

$$
\tilde{\eta}^{2}=\sum_{T} \tilde{\eta}_{T}^{2}
$$

with

$$
\tilde{\eta}_{T}^{2}=|T|\|\mathbf{f}\|_{0, T}^{2}+\frac{1}{2} \sum_{l \in E_{T}}|l|^{2}\left|\mathbf{J}_{l, t}\right|^{2} .
$$

From Lemma 3.3 and Theorems 3.1 and 3.2 we obtain for $\tilde{\eta}$ analogous estimates as for $\eta$ :

Theorem 3.3. There exist constants $C_{1}$ ana $C_{2}$ such that

$$
\|\varepsilon\|_{0}+\left\|\nabla_{k} \mathbf{e}\right\|_{0} \leq C_{1} \tilde{\eta}
$$

and

$$
\tilde{\eta} \leq C_{2}\left\{\|\varepsilon\|_{0}+\left\|\nabla_{k} \mathbf{e}\right\|_{0}+\left(\sum_{T}|T|\left\|\mathbf{f}-\mathbf{f}_{T}\right\|_{0, T}^{2}\right)^{\frac{1}{2}}\right\} .
$$

Remark 3.1. The estimator $\tilde{\eta}$ depends only on the right-hand side $\mathbf{f}$ and the approximate velocity $\mathbf{u}^{k}$, and not on $p^{k}$. Therefore, it can be computed without knowing the approximate pressure. This fact is useful if one computes only $\mathbf{u}^{k}$ by using a divergence-free basis of $\mathbf{V}^{k}$.

Remark 3.2. Following the proof of Lemma 3.1, we see that the part of $\eta$ corresponding to $\mathbf{J}_{l, t}$ does not appear in the bound of that lemma. Therefore, in view of Lemma 3.3, we obtain the better estimate

$$
\|\varepsilon\|_{0} \leq C\left\{\left(\sum_{T}|T|\|\mathbf{f}\|_{0, T}^{2}\right)^{\frac{1}{2}}+\left\|\nabla_{k} \mathbf{e}\right\|_{0}\right\} .
$$

\section{The SECOND-ORDER ElEMENTS OF Fortin AND SOULIE}

We have introduced and analyzed the error estimators only for the lowestdegree nonconforming space for the sake of simplicity. In fact, the first estimator and the results concerning it can be extended straightforwardly to higherorder elements. 
Let us consider the second-order elements of Fortin and Soulie [10]. As was shown in [10], the situation is quite different than in the piecewise linear elements case, since the natural nodes (i.e., the six Gaussian nodes of the sides of $T$ ) cannot be taken as degrees of freedom. The reason is that there exists a quadratic polynomial that vanishes at these six nodes. If $\lambda_{i}$ are the barycentric coordinates of $T$, this polynomial is

$$
p_{T}(\lambda)=2-3\left(\lambda_{1}^{2}+\lambda_{2}^{2}+\lambda_{2}^{2}\right)
$$

Fortin and Soulie showed that the space of piecewise quadratic functions continuous at the two Gaussian nodes of each side coincides with the standard continuous piecewise quadratic elements enriched with an interior node in each triangle associated with $p_{T}$. The computational cost for these elements is essentially the same as that for the standard quadratic elements since the internal degree of freedom can be condensed.

On the other hand, the inf-sup condition is satisfied when these elements are used for the velocity together with discontinuous piecewise linear pressures.

To extend the techniques of $\S 3$ to this case, we observe that the orthogonality relation (3.11) holds also in this case; indeed, denoting with $\llbracket \mathbf{u}^{k} \rrbracket_{l}$ the jump of $\mathbf{u}^{k}$ at $l$, we have

$$
\begin{aligned}
\int_{\Omega} \nabla_{k} \mathbf{e}: \operatorname{curl} \mathbf{s}^{I} & =-\sum_{T} \int_{T} \nabla_{k} \mathbf{u}^{k}: \operatorname{curl} \mathbf{s}^{I} \\
& =-\sum_{T} \int_{\partial T} \mathbf{u}^{k} \cdot\left(\operatorname{curl} \mathbf{s}^{I}\right) \mathbf{n} \\
& =-\sum_{l \in E_{l}} \int_{l} \llbracket \mathbf{u}^{k} \rrbracket_{l} \cdot\left(\operatorname{curl} \mathbf{s}^{I}\right) \mathbf{n}_{l}=0
\end{aligned}
$$

because $\llbracket \mathbf{u}^{k} \rrbracket_{l}$ vanishes at the two Gaussian points of $l$.

To define the estimator, we have to replace the term containing $\|\mathbf{f}\|_{0, T}$ in the piecewise linear case by the local residual (in fact, $f$ was the local residual in that case). Also we have to take into account that $\mathbf{J}_{l, n}$ and $\mathbf{J}_{l, l}$ are not constant in this case. So, we define

$$
\eta_{T}^{2}=|T|\left\|\mathbf{f}+\Delta \mathbf{u}^{k}-\nabla p^{k}\right\|_{0, T}^{2}+\frac{1}{2} \sum_{l \in E_{T}}|l|\left(\left\|\mathbf{J}_{l, n}\right\|_{0, l}^{2}+\left\|\mathbf{J}_{l, l}\right\|_{0, l}^{2}\right) .
$$

The results of Theorem 3.1 and 3.2 hold in this case with $\mathbf{f}_{T}$ replaced by the local $L^{2}$-projection of $\mathbf{f}$ into the linear functions.

Remark 4.1. The proof of Lemma 3.3 cannot be immediately extended to this case. Therefore there is not a straightforward generalization of the estimator $\tilde{\eta}$ to this case.

\section{NUMERICAL RESULTS}

In this section we present the results of numerical computations with the Crouzeix-Raviart elements. We have used $\eta_{T}$ as a local error indicator for adaptive refinement in problems involving singularities. 
The adaptive procedure is as follows. First, we compute $\mathbf{u}^{0}$ and $p^{0}$ corresponding to an initial triangulation $\mathscr{T}^{0}$. Then, the partition $\mathscr{T}^{k+1}$ is obtained from $\mathscr{T}^{k}$ by refining those elements such that

$$
\eta_{T} \geq 0.7 \eta_{\max }
$$

where

$$
\eta_{\max }=\max _{T \in \mathscr{T}^{k}} \eta_{T}
$$

The refinement is propagated using the method introduced in [13]. In this way, the minimum angle of $\mathscr{T}^{k}$ is not less than half of the minimum angle of $\mathscr{T}^{0}$.

In our theoretical results we have assumed, for the sake of simplicity, homogeneous Dirichlet boundary conditions. However, the estimators can be defined with simple modifications for general boundary conditions, and the results are essentially the same. In this case we define $J_{l, n}$ as before and $\mathbf{J}_{l, t}=2\left(\nabla \mathbf{g}^{I}-\nabla \mathbf{u}^{k}\right) \cdot \mathbf{t}_{l}$ if $l \subset \partial \Omega^{*}$, where $\mathbf{g}^{I}$ is the linear interpolation of the Dirichlet boundary datum $\mathbf{g}$ and $\Omega^{*}$ is a polygonal domain approximating $\Omega$. The only difference is the presence of higher-order terms in the equivalence between error and estimator depending on local regularity of the data (see [2, 9] for details in similar situations).

Examples 1 and 2. Let $\Omega=\left\{(r, \varphi): 0<r<1,0<\varphi<\frac{k \pi}{2}\right\}$, with $k=3$ for Example 1 and $k=4$ for Example 2. We solve $-\Delta \mathbf{u}+\nabla p=0$ with homogeneous Dirichlet boundary conditions on the straight parts of the boundary, and nonhomogeneous Dirichlet boundary conditions on the curved part of the boundary. The exact solutions are given by

$$
\begin{gathered}
\mathbf{u}=\left(r^{\alpha}\left[(1+\alpha) \sin \varphi \psi(\varphi)+\cos \varphi \partial_{\varphi} \psi(\varphi)\right],\right. \\
\left.r^{\alpha}\left[\sin \varphi \partial_{\varphi} \psi(\varphi)-(1+\alpha) \cos \varphi \psi(\varphi)\right]\right), \\
p=-r^{\alpha-1}\left[(1+\alpha)^{2} \partial_{\varphi} \psi(\varphi)+\partial_{\varphi}^{3} \psi(\varphi)\right] /(1-\alpha)
\end{gathered}
$$

with

$$
\begin{aligned}
\psi(\varphi)= & \sin ((1+\alpha) \varphi) \cos (\alpha \omega) /(1+\alpha)-\cos ((1+\alpha) \varphi) \\
& +\sin ((\alpha-1) \varphi) \cos (\alpha \omega) /(1-\alpha)+\cos ((\alpha-1) \varphi), \\
\alpha= & 856399 / 1572864, \quad \omega=3 \pi / 2,
\end{aligned}
$$

for Example 1, and

$$
\begin{aligned}
\psi(\varphi) & =3 \sin (0.5 \varphi)-\sin (1.5 \varphi), \\
\alpha & =0.5, \quad \omega=2 \pi,
\end{aligned}
$$

for Example 2.

Table 1 shows the error $\left\|\nabla_{k} e\right\|_{0}+\|\varepsilon\|_{0}$ and the estimator for five steps of the refinement procedure for Example 1. The integer $N$ stands for the number of unknowns. Table 2 shows similar results for Example 2. 
TABLE 1

\begin{tabular}{|rrcr|}
\hline$k$ & \multicolumn{1}{c}{$N$} & $\eta$ & Error \\
\hline 0 & 112 & 2.11 & 5.30 \\
1 & 242 & 1.93 & 3.91 \\
2 & 388 & 1.77 & 3.07 \\
3 & 502 & 1.71 & 2.63 \\
4 & 744 & 1.54 & 2.10 \\
5 & 1220 & 1.39 & 1.55 \\
\hline
\end{tabular}

TABLE 2

\begin{tabular}{|rrrr|}
\hline$k$ & \multicolumn{1}{c}{$N$} & $\eta$ & Error \\
\hline 0 & 148 & 3.55 & 10.74 \\
1 & 302 & 3.38 & 8.53 \\
2 & 592 & 3.06 & 6.32 \\
3 & 834 & 2.82 & 5.01 \\
4 & 1076 & 2.66 & 4.25 \\
5 & 1630 & 2.36 & 3.16 \\
\hline
\end{tabular}

From these results, it follows that optimal order of convergence is obtained for these singular solutions, i.e.,

$$
\|\varepsilon\|_{0}+\left\|\nabla_{k} e\right\|_{0}=\mathscr{O}\left(N^{-1 / 2}\right) .
$$

This is shown in Fig. 1. This order of convergence is the same as that obtained for regular solutions with uniform refinement.

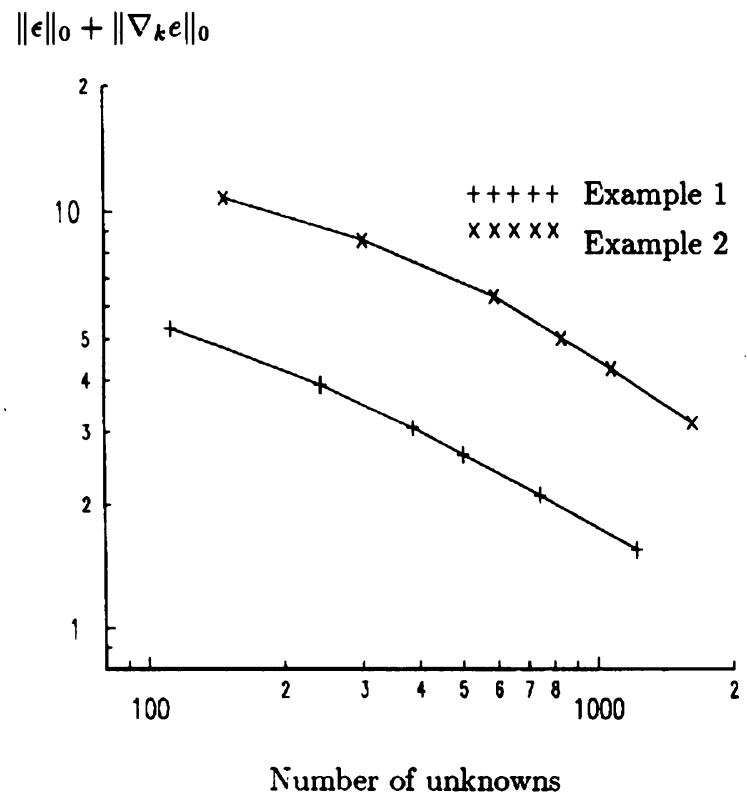

FIGURE 1

Figure 2 shows the meshes $\mathscr{T}^{k}$ for $k=0,1,3,5$ obtained in Example 1 . Figure 3 shows the same meshes for Example 2.

Example 3. This last example is the standard square lid driven cavity. Table 3 shows the estimator for five steps of the refinement procedure.

The results in Table 3 show that $\eta=\mathscr{O}\left(N^{-1 / 2}\right)$, which, according to the theorems proved above guarantees the optimal order of convergence also for this problem. The meshes $\mathscr{T}^{k}$ for $k=0,1,3,5$ obtained in this example are shown in Fig. 4. 

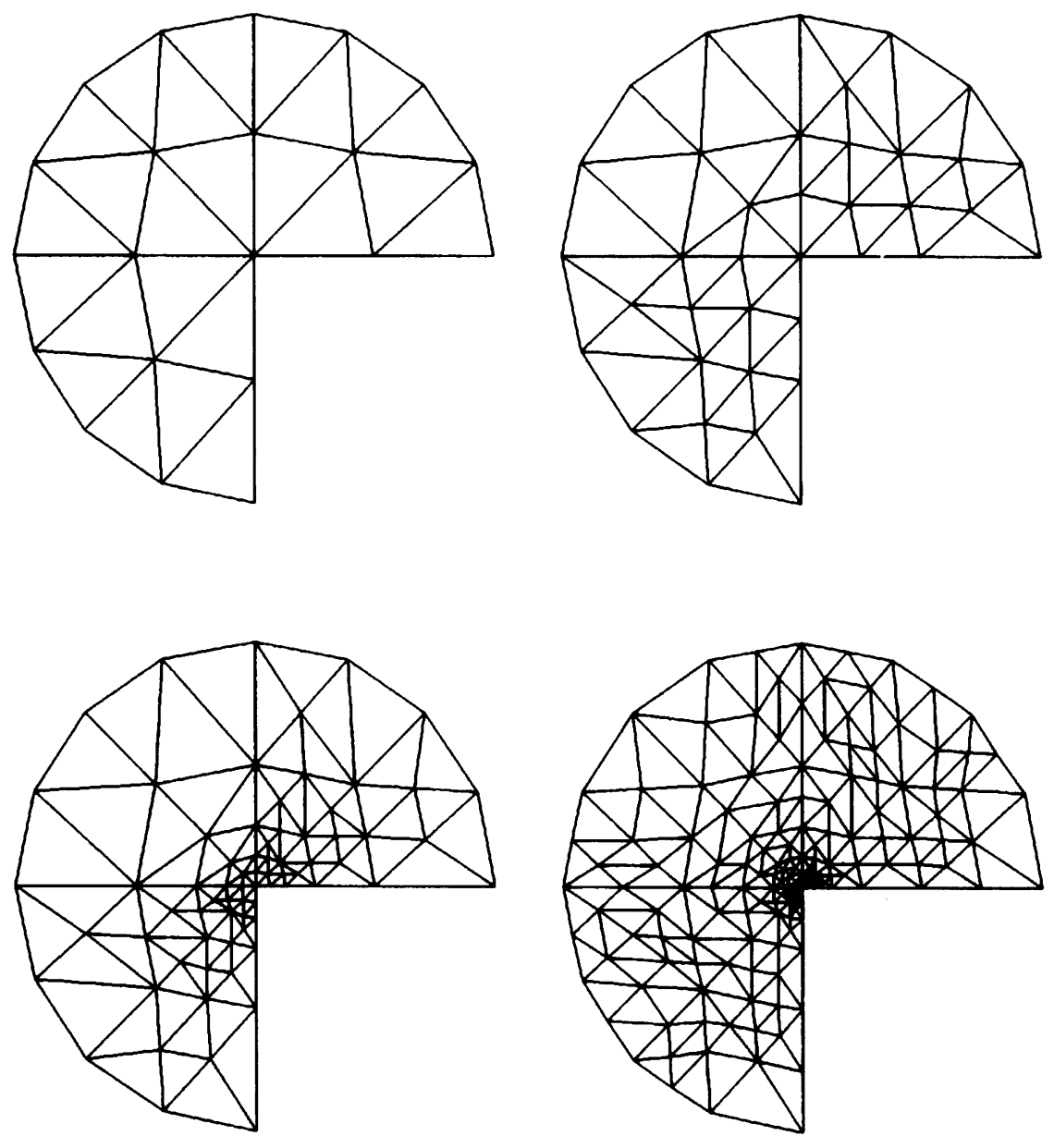

Figure 2 

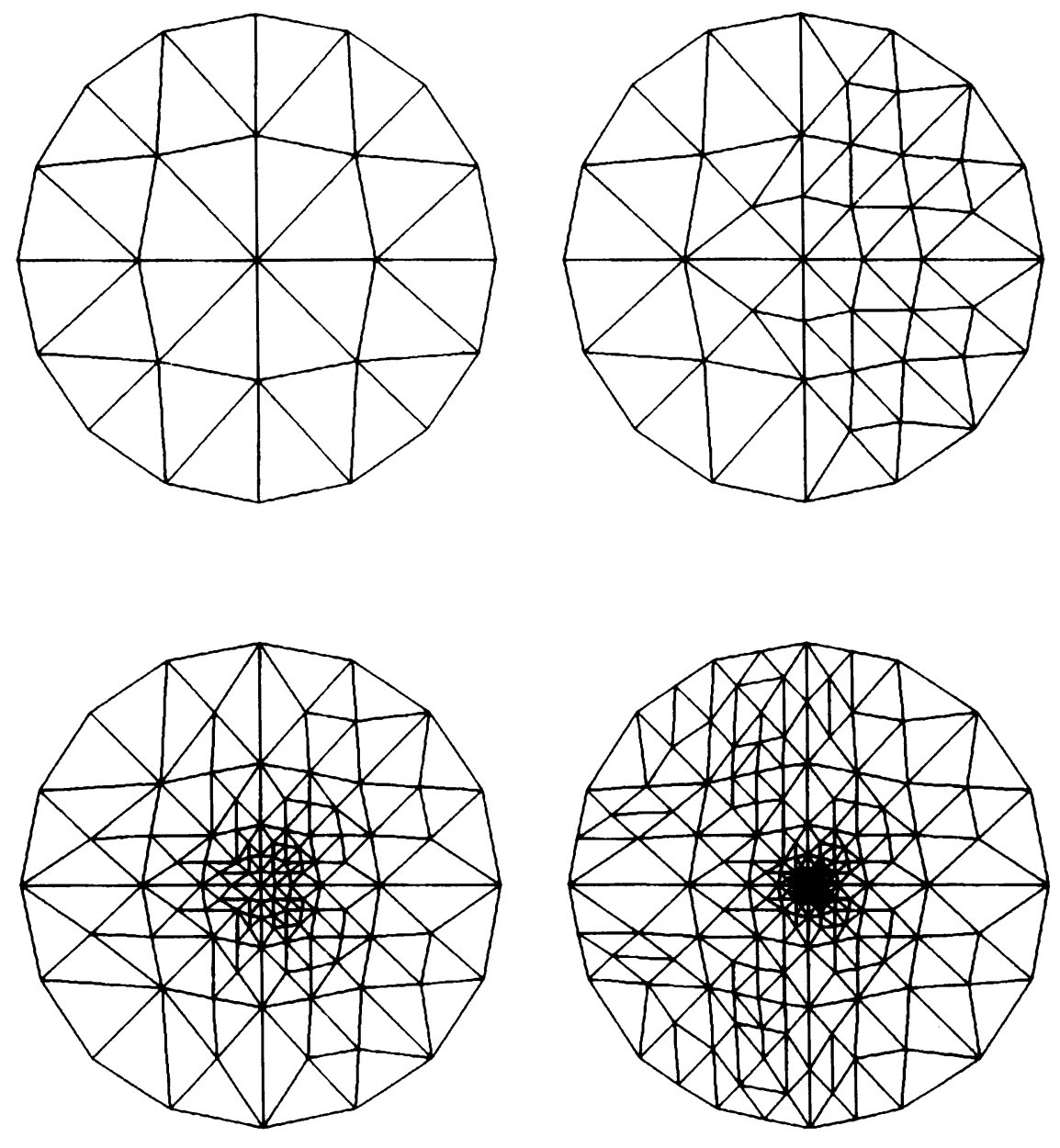

FIGURE 3 
TABLE 3

\begin{tabular}{|rrr|}
\hline$k$ & \multicolumn{1}{c}{$N$} & $\eta$ \\
\hline 0 & 198 & 1.50 \\
1 & 362 & 1.26 \\
2 & 726 & 0.99 \\
3 & 1176 & 0.83 \\
4 & 1616 & 0.72 \\
5 & 2280 & 0.62 \\
\hline
\end{tabular}
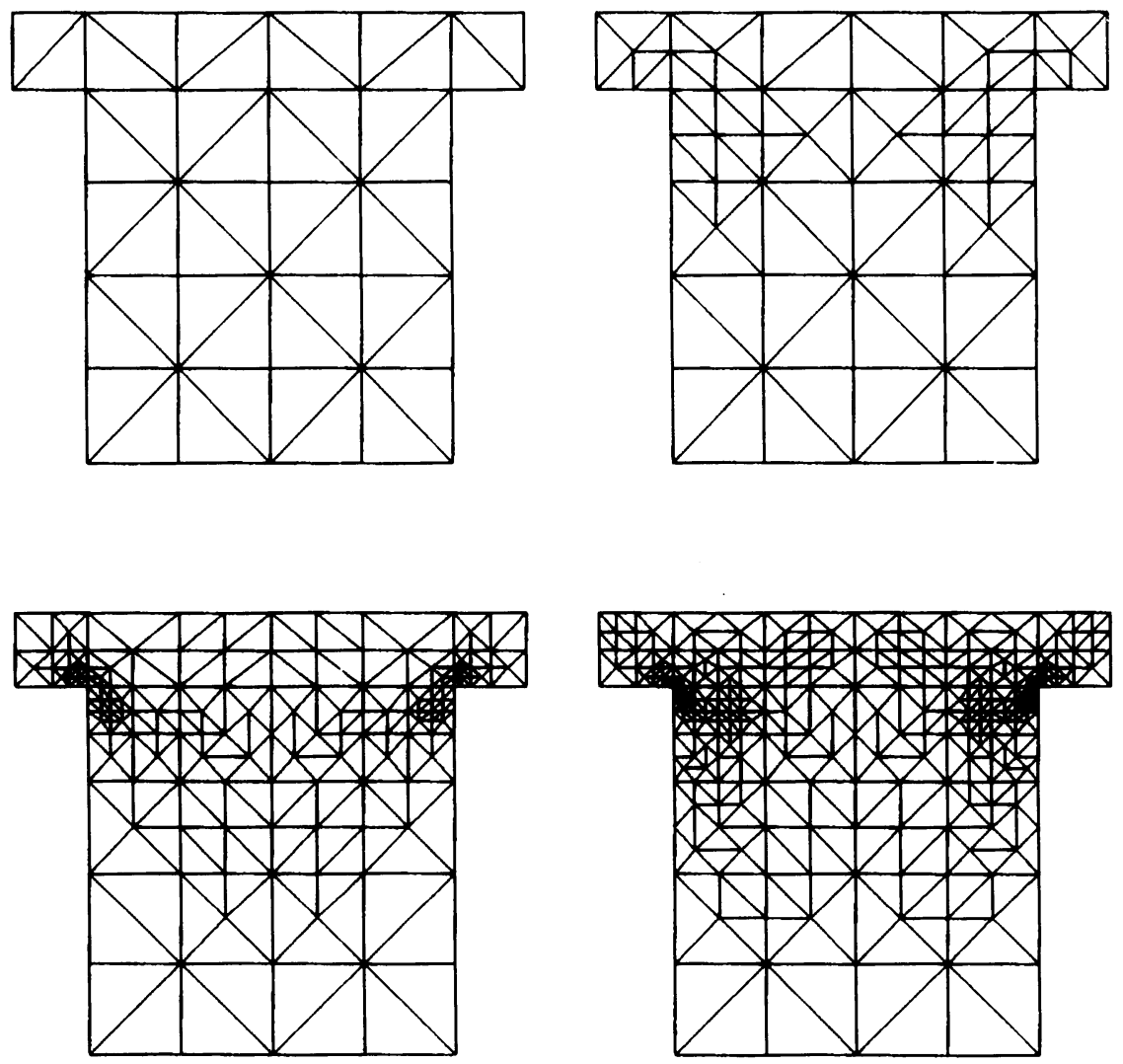

FIGURE 4

\section{CONCLUDING REMARKS}

We have introduced and analyzed a posteriori error estimators for nonconforming approximations of the Stokes problem.

For the case of piecewise linear elements of Crouzeix and Raviart we defined two estimators which are equivalent to the error. Both estimators are easy to compute. However, the second is simpler and depends only on the right-hand side and the approximate velocity; therefore, it can be used when one computes only the velocity by using a divergence-free basis. 
Our numerical computations show good behavior of our first estimator when used as an error indicator for adaptive refinement.

Also, we showed how the first estimator can be generalized for the quadratic elements of Fortin and Soulie. As is easily seen, it can also be generalized for the method of Stenberg and Baroudi [15] in which one component of the velocity is approximated with nonconforming linear elements and the other with standard conforming linear elements. For the same triangulation, the total number of degrees of freedom for these elements is lower than for the Crouzeix and Raviart elements. These two elements have the additional advantage that they can also be used for the elasticity equations.

\section{ACKNOWLEDGMENT}

This work was partially supported by Consejo Nacional de Investigaciones Científicas y Técnicas (CONICET, Argentina) under PID-BID Nos. 39 and 40.

\section{BIBLIOGRAPHY}

1. D. N. Arnold and R. S. Falk, A uniformly accurate finite element method for the Reissner-Mindlin plate, SIAM J. Numer. Anal. 26 (1989), 1276-1290.

2. I. Babuška, R. Durán, and R. Rodriguez, Analysis of the efficiency of an a posteriori error estimator for linear triangular finite elements, SIAM J. Numer. Anal. 29 (1992), 947-964.

3. I. Babuška and A. D. Miller, $A$ feedback finite element method with a posteriori error estimation. Part I: The finite element method and some basic properties of the a posteriori error estimator, Comput. Methods Appl. Mech. Engrg. 61 (1987), 1-40.

4. I. Babuška and W. C. Rheinboldt, A posteriori error estimators in the finite element method, Internat. J. Numer. Methods Engrg. 12 (1978), 1587-1615.

5. R. E. Bank and A. Weiser, Some a posteriori error estimators for elliptic partial differential equations, Math. Comp. 44 (1985), 283-301.

6. R. E. Bank and B. Welfert, A posteriori error estimates for the Stokes equations: a comparison, Comput. Methods Appl. Mech. Engrg. 82 (1990), 323-340.

7. $591-623$

8. M. Crouzeix and P.-A. Raviart, Conforming and nonconforming finite element methods for solving the stationary Stokes equations, RAIRO Anal. Numér. Ser. Rouge (3) 7 (1973), 33-76.

9. E. A. Dari, R. Durán, C. Padra, and V. Vampa, A posteriori error estimators for nonconforming finite element methods, RAIRO Modél. Math. Anal. Numér. (to appear).

10. M. Fortin and M. Soulie, A non-conforming piecewise quadratic finite element on triangles, Internat. J. Numer. Methods Engrg. 19 (1983), 505-520.

11. V. Girault and P.-A. Raviart, Finite element methods for Navier-Stokes equations, SpringerVerlag, Berlin, Heidelberg, 1986.

12. A. K. Noor and I. Babuška, Quality assessment and control of finite element solutions, Finite Elem. in Anal. \& Design 3 (1987), 1-26.

13. M. C. Rivara, Mesh refinement processes hased on the generalized bisection of simplices, SIAM J. Numer. Anal. 21 (1984), 604-613.

14. L. R. Scott and S. Zhang, Finite element interpolation of nonsmooth functions satisfying boundary conditions, Math. Comp. 54 (1990), 483-493. 
15. R. Stenberg and D. Baroudi, $A$ new nonconforming finite element method for incompressible elasticity, Proc. IVth Finnish Mechanics Days, Lappeenranta, June 5-6, 1991 (to appear).

16. R. Verfürth, A posteriori error estimators for the Stokes equations, Numer. Math. 55 (1989), 309-325.

17. _ A posteriori error estimu $\ldots$ ors for the Stokes equations II, non-conforming discretizations, Numer. Math. 60 (1991), 235-249.

(Dari and Padra) Centro Atómico Bariloche, 8400 San Carlos de Bariloche, Río Negro, ARGENTINA

E-mail address, E. Dari: enzo@arib51.bitnet

(Durán) Departamento de Matemática, Facultad de Ciencias Exactas, Universidad de Buenos Aires, 1428 Buenos Aires, Argentina

E-mail address, R. Duran: rduran@mate.dm.uba .ar 